\title{
Laparoscopy Approach to Sigmoid Colon Perforation Secondary to Intrauterine Device Migration
}

Henry Leonardo Robayo Amortegui ( $\square$ henryrobayoamortegui@gmail.com )

Clínica Infantil Colsubsidio https://orcid.org/0000-0003-4613-1703

Diana Marcela Rincon Vanegas

Clinica Infantil Colsubsidio

Adolfredo Ballestas Blanquicet

Clinica Infantil Colsubsidio

Jair Ruiz Fonseca

Clinica Infantil Colsubsidio

\section{Short Communication}

Keywords: Intrauterine Device, Perforation, Sigmoid Colon, Laparoscopy

Posted Date: March 26th, 2020

DOI: https://doi.org/10.21203/rs.3.rs-18906/v1

License: (a) (i) This work is licensed under a Creative Commons Attribution 4.0 International License. Read Full License 


\section{Abstract}

Background Currently, the intrauterine device (IUD) is one of the most used contraceptive methods worldwide, since it has shown to be effective and reversible. One of its complications is the migration of the device, which can affect close organs by perforating them.

Case We present the case of a patient who presented a colouterine fistula secondary to an IUD migration.

Conclusion Laparoscopy is a surgical method that may be performed electively; it makes device removal and repair of affected organs possible with fewer complications.

\section{Introduction}

According to data from the World Health Organization (WHO), the intrauterine device (IUD) is the third most used contraceptive method worldwide, and the fifth most used in Latin America and the Caribbean. It has proved to be a safe, effective and reversible contraceptive method $(1,2)$. Among the rare but serious complications it has been associated with, there is uterine perforation, which can cause lesions in adjacent organs, such as the colon and the small intestine (3).

We present the case of a patient who was scheduled for IUD removal. However, diagnostic laparoscopy showed a colouterine fistula secondary to the migration of the device.

\section{Case Report}

A 23-year-old woman with a history of Rheumatoid Arthritis (RA) who had been using a copper IUD as a contraceptive method for two years. She attended general consultation for pelvic pain and intense dysmenorrhea that had started 3 months earlier. Transvaginal ultrasound reported an intramyometrial IUD with a fragmented aspect. She was evaluated by the gynaecological department, where direct speculoscopy revealed the IUD threads. However, the attempt to retrieve the device was unsuccessful due to patient's pain.

The patient also had a yellow foul-smelling vaginal discharge, so she was given a course of 15-days antibiotic treatment for pelvic inflammatory disease (PID). Due to the previous findings, she underwent hysteroscopy. The threads of the device were not visible, thus, there was a high chance of device migration outside the uterine cavity. We elected to pursue then a diagnostic laparoscopy.

During the procedure, the uterus and adnexa showed normal characteristics, with multiple sigmoid colon adhesions on the posterior wall of the uterus. The general surgeon intervened and performed lysis of adhesions and blunt dissection between the uterus and the sigmoid colon, which eased movement of these two structures and revealed a colouterine fistula (Fig. 1).

The device was completely retrieved (Fig. 2). Suture and perforation closure were performed with intracorporeal 3 - 0 polydioxanone knots (PDS) (Figs. 3 and 4). We cauterized the uterus perforation with 
bipolar forceps and we checked for haemostasis. Knots were tied layer by layer of the skin.

The patient was discharged after two postoperative days; she presented no associated complications. From day one, she had adequate tolerance in her oral tract and had a positive intestinal tract. Her followup evaluation by gynecology and general surgery departments evidenced no latter postoperative complications.

\section{Discussion}

The intrauterine device is a safe contraceptive method, with a $99 \%$ of effectiveness and widely used worldwide (4). Nonetheless, one of its more serious complications is the uterine perforation that occurs with an incidence of 1.6 to 2.1 per 1000 insertions (3). Perforations are reported to be mostly located in the myometrium and the greater omentum, with a frequency of $21 \%$. In the second place, the colon sigmoid with a frequency of $17 \%$ and the left uterosacral ligament and bladder with a $10 \%$ frequency (5). Risk factors for perforation include IUD insertion during lactation, first time using the IUD, insertion during the first 6 months of postpartum, and anatomic abnormalities, such as cervical stenosis and a retroverted uterus $(4,5)$.

In terms of its clinical presentation, up to $85 \%$ of the perforation cases may be asymptomatic (6). However, a 5-year follow-up research carried out in Europe by Barnet et al. found that patients diagnosed with perforation after 12 months of IUD insertion had less probabilities of presenting symptoms. Additionally, the same research revealed that the main symptoms were bleeding and pain (3). In this case, our patient was asymptomatic for two years; she eventually attended consultation for pelvic pain and dysmenorrhea and the transvaginal ultrasound showed a fragmented and intramyometrial IUD, thus, we decided to perform a hysteroscopy to retrieve the device.

According to the WHO guidelines, a fragmented or migrated IUD must be removed as soon as possible, regardless its location (7). The most frequent surgical procedure is laparoscopy, which is also considered first treatment option. However, its success rate varies between $44 \%$ and $100 \%$, depending on the presented complication and the surgeon's expertise. Up to $25 \%$ may have the necessity of conversion to laparotomy $(3,6,8)$.

In our case, we were not able to retrieve the device through hysteroscopy; for that reason, we had to perform a laparoscopy that revealed a colouterine fistula (Fig. 1). We performed removal of the intraperitoneal IUD, we sutured, and we repaired the perforated sigmoid colon (Fig. 4). The patient's 2-day postoperative course had no early or latter complications. The advantages of performing this technique include removal of the device found in the peritoneal cavity, repair through intracorporeal suture, trauma reduction in the affected tissue, pain lessening and reduction of postoperative observation times (9).

\section{Conclusion}


The migration of an IUD may be asymptomatic for months and years; its finding might be even incidental. It must be retrieved as soon as the diagnosis is confirmed. Finally, laparoscopy is a surgical method that may be performed electively; it makes device removal and repair of affected organs possible.

\section{Declarations}

\section{Authors' contributions:}

HRA, DMRV, ABB, JRF: Conception, patient revision, literature review and first draft of the manuscript.

HRA, DMRV: article review, literature review.

HRA, DMRV, ABB, JRF: Article review and Final publication. All the authors read and approved the final manuscript.

\section{Declaration:}

Ethics approval and consent to participate: Approval for publication was obtained from the local ethics committee of Clínica Infantil de Colsubsidio.

Consent for publication: Written informed consent was obtained from the patient's legal guardian(s) for publication of this case report and any accompanying images. A copy of the written consent is available for review by the Editor-in-Chief of this journal.

Availability of data and material: Not applicable.

Competing interests: The authors report no competing interest.

Funding: The authors were completely responsible for the funding of this research.

Acknowledgements: Not applicable

\section{References}

1. United Nations. Contraceptive Use by Method 2019: Data Booklet. Department of Economic and Social Affairs, Population Division; 2019.

2. Mosher WD, Jones J. Use of contraception in the United States: 1982- 2008. Vital Health Stat. 2010;29:1-44.

3. Barnett C, Moehner S, Do Minh T, Heinemann K. Perforation risk and intra-uterine devices: results of the EURAS-IUD 5-year extension study. The European Journal of Contraception \& Reproductive Health Care. 2007;22:6, 424-428. DOI: 10.1080/13625187.2017.1412427.

4. Ti AJ, et al. Effectiveness and safety of extending intrauterine device duration: a systematic review. Am J Obstet Gynecol. 2020 Jan 15. pii: S0002-9378(20)30021-1. doi: 10.1016/j.ajog.2020.01.014. 
5. Sun X, et al. Clinical characteristic and intraoperative findings of uterine perforation patients in using of intrauterine devices (IUDs). Gynecol Surg. 2018;15(1):3. doi:10.1186/s10397-017-1032-2.

6. Toumi 0 , et al. Pelvic abscess complicating sigmoid colon perforation by migrating intrauterine device: A case report and review of the literature. Int J Surg Case Rep. 2018;42:60-63. doi:10.1016/j.jjscr.2017.10.038

7. World Health Organization (WHO). Mechanism of action, safety and efficacy of intrauterine devices. WHO Technical Report 753. Geneva: WHO; 1987.

8. Mosley FR, Shahi N, Kurer MA. Elective surgical removal of migrated intrauterine contraceptive devices from within the peritoneal cavity: a comparison between open and laparoscopic removal. JSLS. 2012;16(2):236-241. doi:10.4293/108680812×13427982377265

9. Weerasekera A, Wijesinghe P, Nugaduwa N. Sigmoid colocolic fistula caused by intrauterine device migration: a case report. J Med Case Rep. 2014;8:81.. doi:10.1186/1752-1947-8-81

\section{Figures}

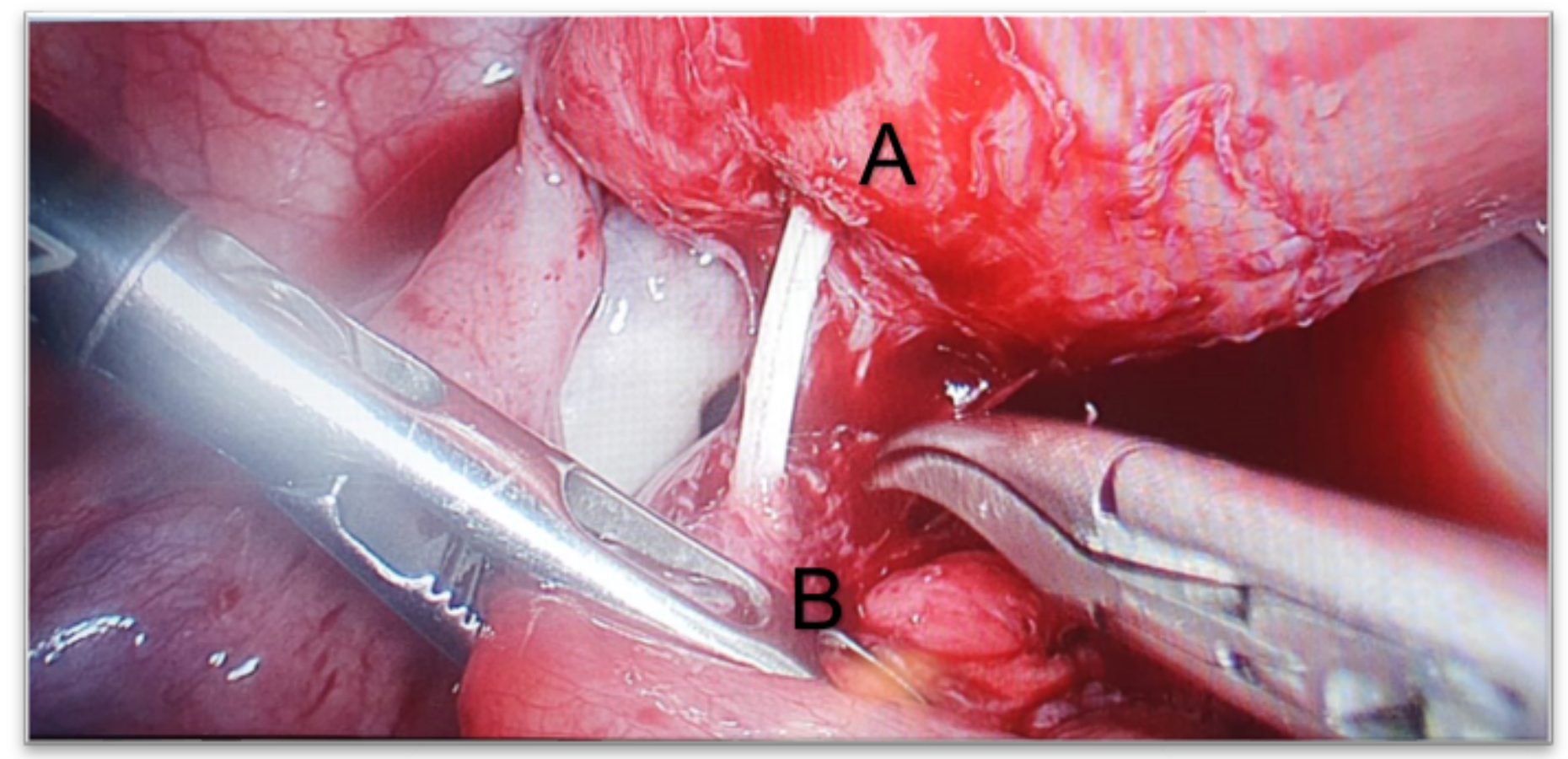

\section{Figure 1}

Colouterine Fistula by IUD. A. Back side of the uterus. B. Sigmoid Colon. 


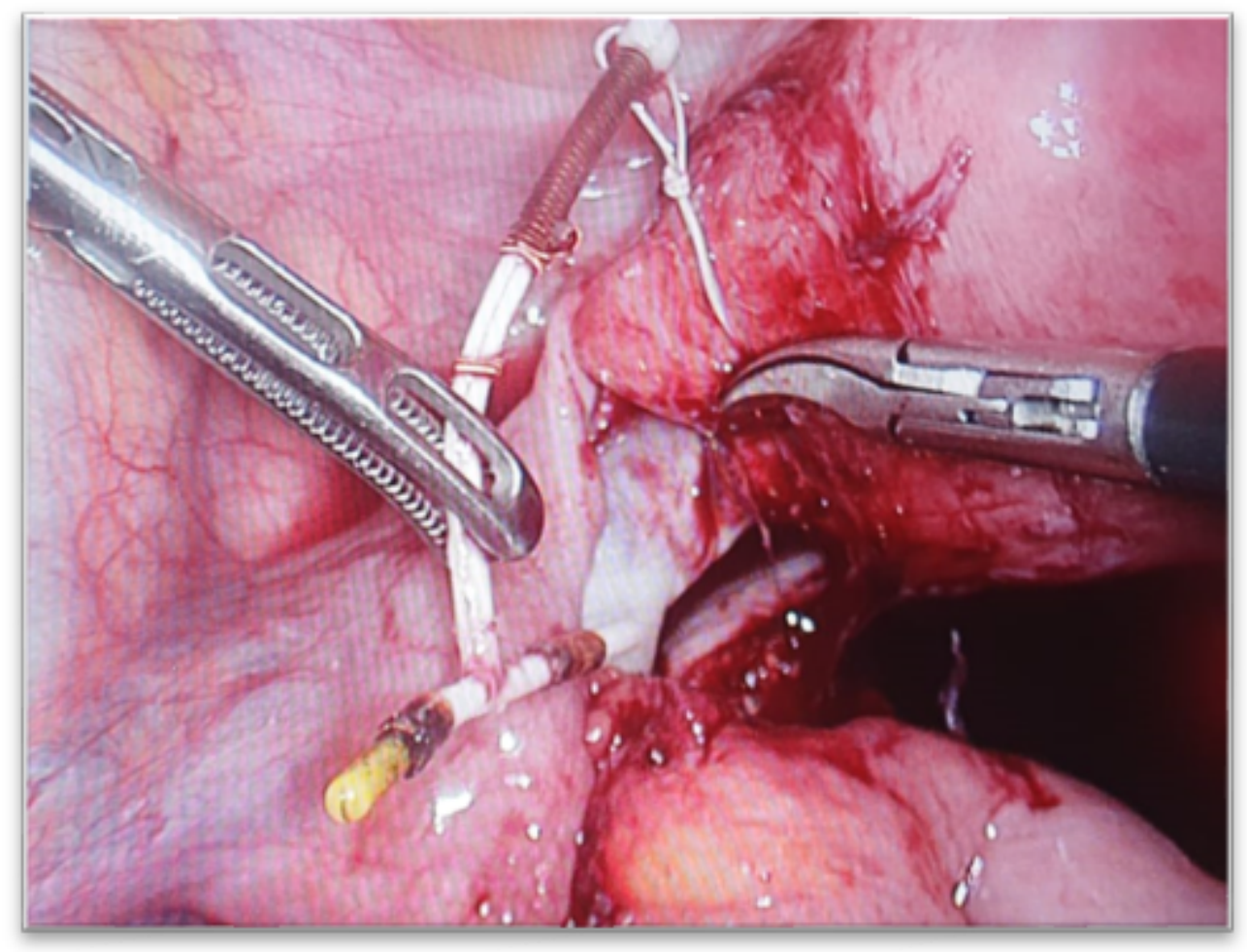

Figure 2

Copper IUD found in the peritoneal cavity. 